\title{
A mechanical model for FRP-strengthened beams in bending
}

\author{
S. Bennati, N. Dardano, P. S. Valvo \\ Università di Pisa, Dipartimento di Ingegneria Civile - Strutture, Largo Lucio Lazzarino, 56126 Pisa \\ s.bennati@ing.unipi.it,p.valvo@ing.unipi.it
}

\begin{abstract}
We analyse the problem of a simply supported beam, strengthened with a fibre-reinforced polymer (FRP) strip bonded to its intrados and subjected to bending couples applied to its end sections. A mechanical model is proposed, whereby the beam and FRP strip are modelled according to classical beam theory, while the adhesive and its neighbouring layers are modelled as an interface having a piecewise linear constitutive law defined over three intervals (elastic response - softening response - debonding). The model is described by a set of differential equations with appropriate boundary conditions. An analytical solution to the problem is determined, including explicit expressions for the internal forces, displacements and interfacial stresses. The model predicts an overall non-linear mechanical response for the strengthened beam, ranging over several stages: from linearly elastic behaviour to damage, until the complete detachment of the FRP reinforcement.
\end{abstract}

SOMMARIO. Nel presente lavoro affrontiamo il problema di una trave semplicemente appoggiata, rinforzata con una striscia di materiale fibro-rinforzato a matrice polimerica (FRP) incollata all'intradosso, e soggetta a coppie flettenti applicate alle sue estremità. Nel modello meccanico proposto, la trave e la striscia di rinforzo sono modellate secondo la teoria delle travi classica, mentre l'adesivo e gli strati adiacenti sono modellati come un'interfaccia avente una legge costitutiva lineare a tratti definita su tre intervalli (risposta elastica - risposta softening - debonding). Il modello è descritto da un sistema di equazioni differenziali, completato da opportune condizioni al contorno, che viene risolto analiticamente ricavando espressioni esplicite per le principali grandezze di interesse (spostamenti, caratteristiche della sollecitazione, sforzi d'interfaccia). Il modello prevede per la trave rinforzata una risposta meccanica complessiva non lineare, articolata in più fasi: da quella elastica lineare a quella danneggiata, fino ad arrivare al completo distacco del rinforzo di FRP.

KEYWORDS. FRP strengthening; Adhesive; Beam theory; Interface.

\section{INTRODUCTION}

$\mathrm{F}$ ibre-reinforced polymers (FRP) are currently used in civil engineering to carry out strengthening and repair operations on existing constructions. The pre-existing structural elements (made of traditional materials such as, for instance, masonry, wood, concrete, steel etc.) are strengthened by gluing FRP reinforcement sheets or strips on their external surface. The shape and thickness of the external reinforcement obviously vary according to the type of element and the level of structural performance desired [1-4].

In order to evaluate the effectiveness of such operations, however, it is necessary to take in account the possible emergence of phenomena of delamination or detachment of the FRP reinforcement, which can seriously jeopardize the outcome of the strengthening operations. Moreover, delamination and detachment phenomena may manifest themselves in various and complex ways. In the case of concrete structures, for instance, delamination may occur at different levels involving the FRP strips themselves, the adhesive and/or the superficial layers of the concrete. Delamination is caused 
mainly by the stresses that develop on the interface between the bonded elements. In order to account for these possible effects, various theoretical models have been developed that enable evaluating such stresses. Moreover, a number of special laboratory tests have been studied to evaluate the strength of the bonding under different loading conditions.

One very commonly adopted test procedure is to glue an FRP strip to a block of concrete and then subject it to tension until it detaches completely [5,6]. Various theoretical models have been proposed for this test and differ mainly in the constitutive law assumed for the interface between the concrete and FRP. In general, the different models assume an initially linear elastic response, in which the stresses tangential to the interface are proportional to the corresponding relative displacements. Subsequently, the models assume a softening response, which is meant to represent the progressive damage occurring prior to complete detachment of the reinforcement. Cottone and Giambanco [7] have formulated a linear decreasing law for the softening phase, while Cornetti and Carpinteri [8] propose an exponential decreasing one.

In the present work we address the problem of a simply supported beam, strengthened with an FRP strip bonded to its intrados and subjected to bending couples applied to its end sections. A similar problem, though with the concentrated load applied to the mid-span section, has been considered by Carpinteri et al. [9] and by De Lorenzis and Zavarise [10]. In the mechanical model proposed here, the beam and reinforcement strip are bonded by a zero-thickness interface representing the adhesive layer and the superficial layers of the bonded elements. The interface transfers tangential stresses only from one element to the other. Furthermore, the following simplifying hypotheses are adopted:

a) the beam is flexible, but inextensible, and exhibits indefinitely elastic behaviour;

b) the reinforcement strip is extensible, but completely lacking flexural rigidity, hence it is subject to solely axial stresses; c) the tangential stresses transferred by the interface are functions of the relative displacements between the beam and the strip through a piecewise linear function defined over three intervals (elastic response - softening response - debonding). The mechanical model is described by a set of differential equations, supplemented by appropriate boundary conditions. The resulting mathematical problem is solved analytically and explicit expressions for the main quantities of interest (displacements, internal forces, interface stresses) are determined.

The model predicts an overall non-linear mechanical response for the FRP-strengthened beam, which passes through various distinct, recognisable stages of behaviour as the intensity of the applied bending couples is increased. In the first stage, the entire system exhibits a linearly elastic response and the beam can be divided into two parts: the portion initially lacking reinforcement and that connected to the reinforcement through the interface, which is still completely in the elastic field. The first stage of behaviour ends when the tangential interfacial stresses at the extremities of the reinforcement reach the elastic limit. In the second stage, the interface portions near the reinforcement extremities enter the softening response domain and the beam is thus divided into three parts: the portion initially lacking reinforcement and those portions connected to the reinforcement through the interface, which is now partly damaged and partly still in the elastic field. The second stage of behaviour ends when the relative displacements at the extremities of the reinforcement reach their ultimate value, corresponding to interface debonding. In the third stage, the reinforcement strip progressively detaches from the beam, beginning at the extremities, and the beam can be thought of as still divided into three parts: an unreinforced portion made up of the area initially lacking reinforcement plus the area with the now detached strip, a portion whose reinforcement is connected via the damaged interface and the portion with reinforcement still connected by an elastic interface. As the loads increase, the damaged and debonded interface portions extend to involve the entire length of the beam. Upon complete detachment, the overall response of the system reduces to that of the beam lacking any reinforcement. It is interesting to note that this last stage is however only reached asymptotically with infinite growth of the couples applied to the beam.

\section{MECHANICAL MODEL}

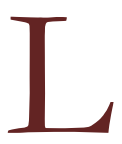
et us consider a beam $A B$ of length $L=2 l$, simply supported at its ends, where it is loaded by two equal and opposite couples of magnitude $\bar{M}$ (Fig. 1). A strip of FRP reinforcement is bonded along the centre of the beam intrados for a length $2 b_{0}$. We indicate $H$ as the height of the cross section of the beam and $h$ the distance of the section's centre from its intrados (for a rectangular cross section, $h=H / 2$ ).

Thanks to the symmetry of the problem, it is possible to limit the study to the left half alone by introducing appropriate constraints in correspondence to the axis of symmetry (Fig. 2). The positions of the beam and strip cross sections are given by an abscissa $s$, whose origin is placed at the left-hand extremity of the active (not detached) portion of the reinforcement strip. This choice helps obtaining simpler expressions for the integration constants characterising the analytical solution. With $a_{0}=l-b_{0}$ the length of the initially unreinforced segment of the beam, we indicate by $v(s)$ and 
$\phi(s)$ the transverse displacement and rotation (positive if clockwise) of the cross section of the beam, respectively, and by $w(s)$ the axial displacement of the cross section of the reinforcement.

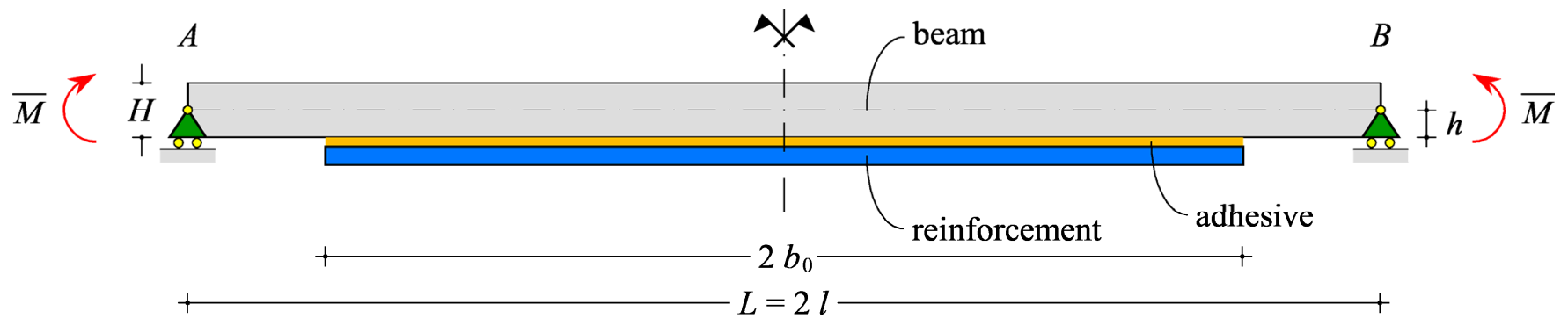

Figure 1: FRP-strengthened beam in bending.

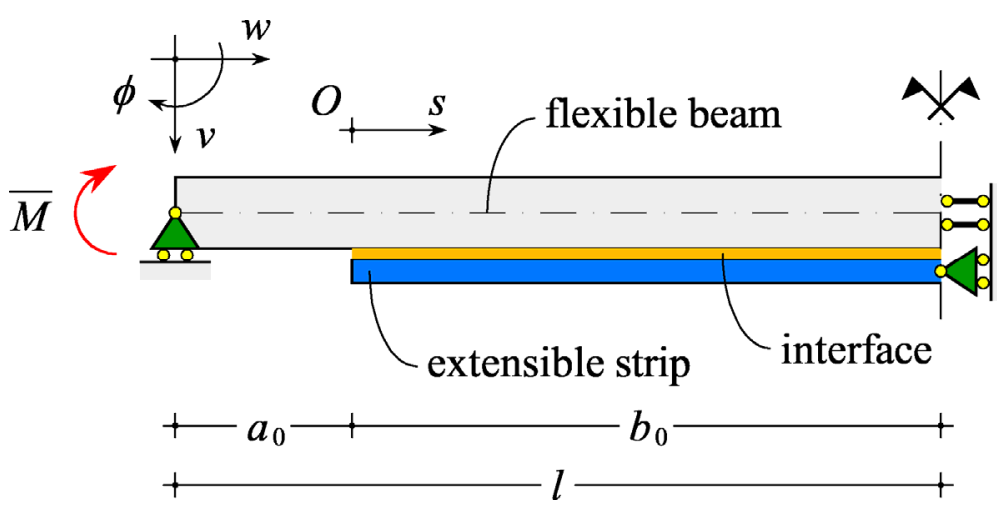

Figure 2: Mechanical model of the strengthened beam.

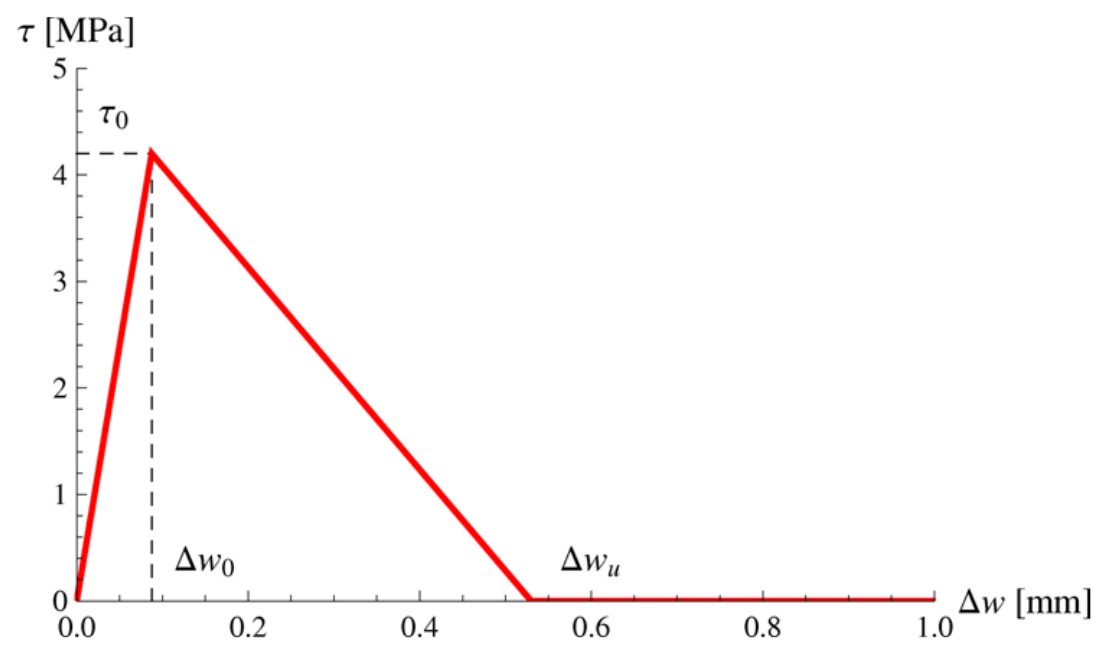

Figure 3: Constitutive law of the interface.

In the proposed mechanical model, the beam is flexible, but inextensible, while the reinforcement strip is extensible only. We denote $E$ and $J$ as the Young's modulus and moment of inertia of the cross section of the beam, respectively, and $E_{f}$ and $A_{f}$ as the Young's modulus and area of the cross section of the FRP strip, respectively. With $B_{f}$ the width of the reinforcement strip, and $t_{f}$ its thickness, we have $A_{f}=B_{f} t_{f}$. The beam and reinforcement are connected to each other by an interface of negligible thickness. Since the reinforcement is subject to axial stresses alone, the interface transmits only tangential stresses, $\tau(s)$, which we assume are functions of the relative displacement between beam and reinforcement in correspondence to the interface, 


$$
\Delta w(s)=w(s)+h \phi(s)
$$

In particular, we assume the following piecewise linear constitutive law (Fig. 3):

$$
\tau(\Delta w)=\left\{\begin{array}{lll}
k \Delta w, & 0 \leq \Delta w \leq \Delta w_{0} & \text { (elastic response) } \\
k_{s}\left(\Delta w_{u}-\Delta w\right), & \Delta w_{0}<\Delta w \leq \Delta w_{u} & \text { (softening response) } \\
0, & \Delta w_{u}<\Delta w & \text { (debonding) }
\end{array}\right.
$$

where $k$ and $k_{s}$ are the interface elastic constants for the fields of elastic and softening response, respectively, and $\Delta w_{0}$ and $\Delta w_{u}$ are the relative displacements corresponding, respectively, to the elastic limit and debonding of the interface.

\section{DIFFERENTIAL PROBLEM}

he behaviour of the FRP-strengthened beam varies according to the response field in which the interface is found. The adopted piecewise linear constitutive law enables distinguishing three cases, for which the differential problem is appropriately formulated and solved in the following. The resulting solutions will then be employed to reconstruct the overall response of the system in the different stages of behaviour.

\section{Case a) Interface with elastic response}

In the case of the interface response in the elastic field, from the equilibrium equations and the constitutive law we formulate the following set of governing differential equations (here and henceforth, primes indicate derivation with respect to the abscissa $s$; the quantities in this case are indicated with the subscript $e=$ elastic):

$$
\left\{\begin{array}{l}
v_{e}{ }^{\prime \prime \prime}(s)-\frac{k B_{f} h^{2}}{E J} v_{e}{ }^{\prime}(s)-\frac{k B_{f} h}{E J} w_{e}{ }^{\prime}(s)=0 \\
w_{e}{ }^{\prime \prime}(s)-\frac{k B_{f}}{E_{f} A_{f}} w_{e}(s)-\frac{k B_{f} h}{E_{f} A_{f}} v_{e}{ }^{\prime}(s)=0
\end{array}\right.
$$

By solving Eqs. (3), we obtain the general solution for the transverse displacement of the beam,

$$
v_{e}(s)=A_{1} \cosh \alpha s+A_{2} \sinh \alpha s+A_{3} s^{3}+A_{4} s^{2}+A_{5} s+A_{6}
$$

and for the axial displacement of the reinforcement,

$$
w_{e}(s)=\frac{E J}{E_{f} A_{f} h} \alpha\left(A_{1} \sinh \alpha s+A_{2} \cosh \alpha s\right)-3 A_{3} h s^{2}-2 A_{4} h s-6 A_{3} \frac{E_{f} A_{f} h}{k B_{f}}-A_{5} h
$$

where $\alpha^{2}=k B_{f}\left(1 / E_{f} A_{f}+h^{2} / E J\right)$. Thus, we can obtain the rotation of the beam cross section,

$$
\phi_{e}(s)=v_{e}{ }^{\prime}(s)=\alpha\left(A_{1} \sinh \alpha s+A_{2} \cosh \alpha s\right)+3 A_{3} s^{2}+2 A_{4} s+A_{5}
$$

the relative displacement at the interface,

$$
\Delta w_{e}(s)=w_{e}(s)+h \phi_{e}(s)=\frac{E J}{k B_{f} h} \alpha^{3}\left(A_{1} \sinh \alpha s+A_{2} \cosh \alpha s\right)-6 A_{3} \frac{E_{f} A_{f} h}{k B_{f}}
$$

the tangential stress at the interface,

$$
\tau_{e}(s)=k \Delta w_{e}(s)=\frac{E J}{B_{f} h} \alpha^{3}\left(A_{1} \sinh \alpha s+A_{2} \cosh \alpha s\right)-6 A_{3} \frac{E_{f} A_{f} h}{B_{f}}
$$

the bending moment in the beam, 


$$
M_{e}(s)=-E J v_{e} "(s)=-E J\left[\alpha^{2}\left(A_{1} \cosh \alpha s+A_{2} \sinh \alpha s\right)+6 A_{3} s+2 A_{4}\right]
$$

the shear force in the beam,

$$
T_{e}(s)=-E J v_{e}{ }^{\prime \prime}(s)+h B_{f} \tau_{e}(s)=-6 A_{3}\left(E J+E_{f} A_{f} h^{2}\right)
$$

and, lastly, the axial force in the reinforcement,

$$
N_{e}(s)=E A w_{e}{ }^{\prime}(s)=\frac{E J}{h} \alpha^{2}\left(A_{1} \cosh \alpha s+A_{2} \sinh \alpha s\right)-2 E_{f} A_{f} h\left(3 A_{3} s+A_{4}\right)
$$

In Eqs. (4)-(11), $A_{1}, A_{2}, \ldots, A_{6}$ are integration constants to be determined by imposing boundary conditions.

\section{Case b) Interface with softening response}

In the case of the interface response in the softening field of behaviour, the equilibrium equations and the constitutive law enable us to formulate the following set of governing differential equations (the quantities in this case are indicated by the subscript $s=$ softening ):

$$
\left\{\begin{array}{l}
v_{s}{ }^{\prime \prime \prime}(s)+\frac{k_{s} B_{f} h^{2}}{E J} v_{s}{ }^{\prime}(s)+\frac{k_{s} B_{f} h}{E J} w_{s}{ }^{\prime}(s)=0 \\
w_{s}{ }^{\prime \prime}(s)+\frac{k_{s} B_{f}}{E_{f} A_{f}} w_{s}(s)+\frac{k_{s} B_{f} h}{E_{f} A_{f}} v_{s}{ }^{\prime}(s)-\frac{k_{s} B_{f}}{E_{f} A_{f}} \Delta w_{u}=0
\end{array}\right.
$$

Solving Eqs. (12) yields the general solution for the transverse displacement of the beam,

$$
v_{s}(s)=B_{1} \cos \beta s+B_{2} \sin \beta s+B_{3} s^{3}+B_{4} s^{2}+B_{5} s+B_{6}
$$

and the axial displacement of the reinforcement,

$$
w_{s}(s)=\frac{E J}{E_{f} A_{f} h} \beta\left(-B_{1} \sin \beta s+B_{2} \cos \beta s\right)-3 B_{3} h s^{2}-2 B_{4} h s+6 B_{3} \frac{E_{f} A_{f} h}{k_{s} B_{f}}-B_{5} h+\Delta w_{u}
$$

where $\beta^{2}=k_{s} B_{f}\left(1 / E_{f} A_{f}+h^{2} / E J\right)$. Thus, we can now obtain the rotation of the beam cross section,

$$
\phi_{s}(s)=v_{s}{ }^{\prime}(s)=\beta\left(-B_{1} \sin \beta s+B_{2} \cos \beta s\right)+3 B_{3} s^{2}+2 B_{4} s+B_{5}
$$

the relative displacement at the interface,

$$
\Delta w_{s}(s)=w_{s}(s)+h \phi_{s}(s)=\frac{E J}{k_{s} B_{f} h} \beta^{3}\left(-B_{1} \sin \beta s+B_{2} \cos \beta s\right)+6 B_{3} \frac{E_{f} A_{f} h}{k_{s} B_{f}}+\Delta w_{u}
$$

the tangential stress at the interface,

$$
\tau_{s}(s)=k_{s}\left[\Delta w_{u}-\Delta w_{s}(s)\right]=\frac{E J}{B_{f} h} \beta^{3}\left(B_{1} \sin \beta s-B_{2} \cos \beta s\right)-6 B_{3} \frac{E_{f} A_{f} h}{B_{f}}
$$

the bending moment in the beam,

$$
M_{s}(s)=-E J v_{s} "(s)=E J\left[\beta^{2}\left(B_{1} \cos \beta s+B_{2} \sin \beta s\right)-6 B_{3} s-2 B_{4}\right]
$$

the shear force in the beam,

$$
T_{s}(s)=-E J v_{s}{ }^{\prime \prime}(s)+h B_{f} \tau_{s}(s)=-6 B_{3}\left(E J+E_{f} A_{f} h^{2}\right)
$$

and, lastly, the axial force in the reinforcement, 


$$
N_{s}(s)=E A w_{s}{ }^{\prime}(s)=-\frac{E J}{h} \beta^{2}\left(B_{1} \cos \beta s+B_{2} \sin \beta s\right)-2 E_{f} A_{f} h\left(3 B_{3} s+B_{4}\right)
$$

In Eqs. (13)-(20), $B_{1}, B_{2}, \ldots, B_{6}$ are integration constants to be determined by imposing boundary conditions.

\section{Case c) Debonded interface (or lacking reinforcement)}

In the case that the interface is debonded (or the reinforcement is absent right from the start), the differential equation for the beam is simply (the quantities in this case are indicated by the subscript $d=$ debonding ):

$$
E J v_{d}^{\prime \prime \prime}(s)=0
$$

The transverse displacement of the beam has the following expression

$$
v_{d}(s)=C_{1} s^{3}+C_{2} s^{2}+C_{3} s+C_{4}
$$

Thus, we can obtain the rotation of the beam cross section,

$$
\phi_{d}(s)=v_{d}^{\prime}(s)=3 C_{1} s^{2}+2 C_{2} s+C_{3}
$$

the bending moment in the beam,

$$
M_{d}(s)=-E J v_{d} "(s)=-E J\left(6 C_{1} s+2 C_{2}\right)
$$

and lastly, the shear force in the beam,

$$
T_{d}(s)=-E J v_{d} " '(s)=-6 C_{1} E J
$$

In Eqs. (22)-(25), $C_{1}, C_{2}, C_{3}, C_{4}$ are integration constants to be determined by imposing boundary conditions.

\section{SOLUTION FOR THE FRP-STRENGTHENED BEAM IN THE DIFFERENT STAGES OF BEHAVIOUR}

\section{Stage 1) Entirely elastic interface}

$\mathrm{F}$ or small values of the applied couple $\bar{M}$, the system exhibits a linearly elastic response. In particular, the entire interface is within the initial stage of elastic behaviour. The strengthened beam can be divided into two parts: the portion lacking reinforcement, of length $a_{0}$, and that with the reinforcement, of length $b_{0}=l-a_{0}$ (Fig. 4). In these two distinct regions, the solution to the problem is given by Eqs. (22)-(25) and Eqs. (4)-(11), respectively. Now, imposing the boundary conditions,

$$
\begin{aligned}
& v_{d}\left(-a_{0}\right)=0, \quad M_{d}\left(a_{0}\right)=\bar{M} \\
& v_{d}(0)=v_{e}(0), \quad \phi_{d}(0)=\phi_{e}(0), \quad M_{d}(0)=M_{e}(0), \quad T_{d}(0)=T_{e}(0), \quad N_{e}(0)=0 \\
& \phi_{e}\left(b_{0}\right)=0, \quad T_{e}\left(b_{0}\right)=0, \quad w_{e}\left(b_{0}\right)=0
\end{aligned}
$$

yields the integration constants for stage 1, whose analytical expressions are given in the Appendix.

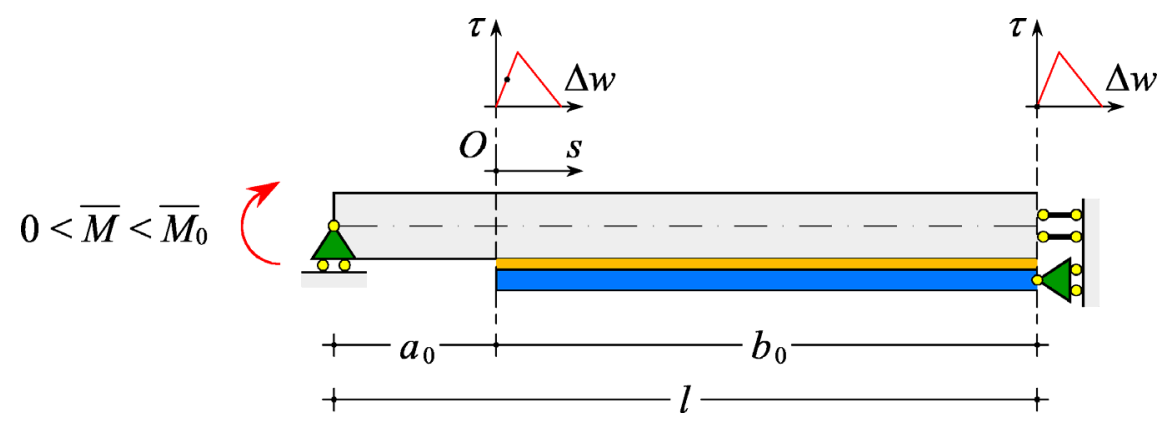

Figure 4: Stage 1) Entirely elastic interface. 
Stage 1 takes place for values of the applied couple $\bar{M}$ between 0 and $\bar{M}_{0}$. To determine the latter value, it is sufficient to consider that stage 1 ends when the (maximum) relative displacement at the extremity of the FRP strip reaches the elastic limit value, that is, when

$$
\Delta w(0)=\Delta w_{0}
$$

By substituting the solution obtained for the relative displacement, $\Delta w$, into Eq. (27), after some simplifications, we get

$$
\bar{M}_{0}=\Delta w_{0} \frac{E J}{h} \alpha \operatorname{coth} \alpha b_{0}
$$

which is the value of the applied couple corresponding to the end of stage 1 . For current applications, the argument of the hyperbolic function appearing in Eq. (28) is quite large, so that the following approximate expression can be used:

$$
\bar{M}_{0} \cong \alpha \frac{E J}{h} \Delta w_{0}
$$

\section{Stage 2) Elastic-Damaged interface}

As the intensity of the applied couple $\bar{M}$ grows beyond the value $\bar{M}_{0}$, the FRP-strengthened beam enters stage 2 of behaviour. The interface response is partly still in the elastic field (elastic interface) and partly in the softening field (damaged interface). The strengthened beam can be divided into three parts: the portion lacking reinforcement, of length $a_{0}$, the portion with the damaged interface, of length $c$ (to be determined), and the portion with the elastic interface, of length $d=b_{0}-c$ (Fig. 5). In these three distinct regions, the solution to the problem is respectively given by Eqs. (22)(25), Eqs. (13)-(20) and Eqs. (4)-(11). Imposing the boundary conditions,

$$
\begin{aligned}
& v_{d}\left(-a_{0}\right)=0, \quad M_{d}\left(-a_{0}\right)=\bar{M} \\
& v_{d}(0)=v_{s}(0), \quad \phi_{d}(0)=\phi_{s}(0), \quad M_{d}(0)=M_{s}(0), \quad T_{d}(0)=T_{s}(0), \quad N_{s}(0)=0 \\
& v_{s}(c)=v_{e}(c), \quad \phi_{s}(c)=\phi_{e}(c), \quad M_{s}(c)=M_{e}(c), \quad T_{s}(c)=T_{e}(c), \quad w_{s}(c)=w_{e}(c), \quad N_{s}(c)=N_{e}(c) \\
& \phi_{e}\left(b_{0}\right)=0, \quad T_{e}\left(b_{0}\right)=0, \quad w_{e}\left(b_{0}\right)=0
\end{aligned}
$$

yields the integration constants for stage 2, whose analytical expressions are given in the Appendix.

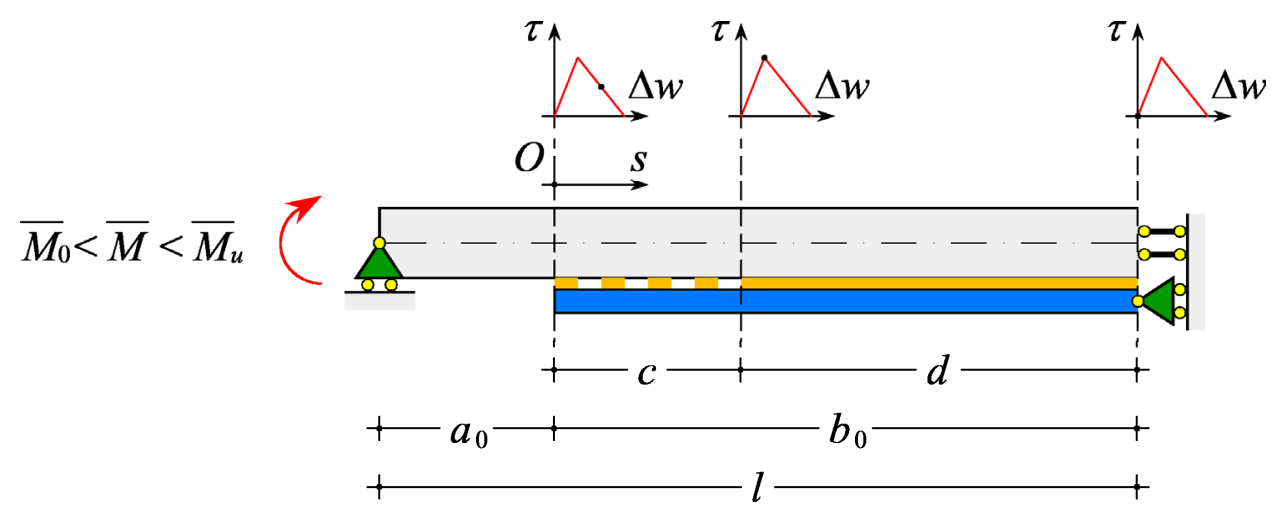

Figure 5: Stage 2) Elastic-Damaged interface.

The unknown length $c$ can be determined by requiring that the relative displacement in the transition section between the damaged and elastic interface portions be equal to the value corresponding to the elastic limit:

$$
\Delta w(c)=\Delta w_{0}
$$

Rendering Eq. (31) explicit and taking into account the expressions for the integration constants listed in the Appendix, we obtain the value of the applied couple corresponding to any given value of $c$ : 


$$
\bar{M}=\bar{M}_{0}\left[\cos \beta c \operatorname{coth} \alpha\left(b_{0}-c\right)+\frac{\alpha}{\beta} \sin \beta c\right] \tanh \alpha b_{0}
$$

Vice versa, by (numerically) solving Eq. (32), we can determine the length $c$ as a function of $\bar{M}$. It can thus be seen that $c$ grows with $\bar{M}$ throughout the entire stage 2 . The value of the applied couple, $\bar{M}_{u}$, at the end of this stage of behaviour is determined by imposing that the (maximum) relative displacement at the extremity of the FRP strip be equal to the value corresponding to interface debonding:

$$
\Delta w(0)=\Delta w_{u}
$$

By rendering Eq. (33) explicit and simplifying, we obtain

$$
\alpha \tanh \alpha\left(b_{0}-c_{u}\right)=\beta \tan \beta c_{u}
$$

Eq. (34) can be solved numerically to determine the maximum value of $c$, indicated by $c_{u}$, corresponding to the end of stage 2. To this purpose, noting that since the first member of Eq. (34) is always positive, the second must be likewise, hence the following condition must hold,

$$
c_{u} \leq c_{c}=\frac{\pi}{2 \beta}
$$

where $c_{c}$ is a characteristic length. The system's response is different in the three cases corresponding to a reinforcement length, $b_{0}$, respectively, less than, equal to, or greater than $c_{c}$. An analogous result has been found by Cottone and Giambanco [7] for the problem of a stretched strip. For our problem, however, considering current parameter values, it is likely that $b_{0}>c_{c}$, so that we will focus on this case only in what follows. Furthermore, the hyperbolic tangent in Eq. (34) takes on values very near unity. Therefore, instead of solving Eq. (34) numerically, it is also possible to use the approximate expression

$$
c_{u} \cong \frac{1}{\beta} \arctan \frac{\alpha}{\beta}
$$

By substituting the calculated value of $c_{u}$ into Eq. (32), we finally obtain the value of the couple, $\bar{M}_{u}$, applied at the end of stage 2. In particular, from the approximate value given by Eq. (36), we get

$$
\bar{M}_{u} \cong \bar{M}_{0} \sqrt{1+\frac{\alpha^{2}}{\beta^{2}}}=\bar{M}_{0} \sqrt{1+\frac{k}{k_{s}}}=\bar{M}_{0} \sqrt{\frac{\Delta w_{u}}{\Delta w_{0}}}
$$

\section{Stage 3) Elastic-Damaged-Debonded interface}

As the intensity of the applied couple $\bar{M}$ increases beyond the value $\bar{M}_{u}$, the FRP-strengthened beam enters stage 3 of behaviour. The interface portions near the reinforcement extremities become debonded and the reinforcement progressively detaches from the beam. In this stage, the beam can once again be divided into three parts: the total unreinforced portion (made up of the part initially lacking reinforcement and the part whose reinforcement has detached), of length $a$ (to be determined), the damaged interface portion, of length $c$ (also to be determined), and the still elastic interface portion, of length $d=l-a-c$ (Fig. 6). In these three distinct regions, the solution to the problem is given respectively by Eqs. (22)-(25), Eqs. (13)-(20) and Eqs. (4)-(11). Imposing the boundary conditions,

$$
\begin{aligned}
& v_{d}(-a)=0, \quad M_{d}(-a)=\bar{M} \\
& v_{d}(0)=v_{s}(0), \quad \phi_{d}(0)=\phi_{s}(0), \quad M_{d}(0)=M_{s}(0), \quad T_{d}(0)=T_{s}(0), \quad N_{s}(0)=0 \\
& v_{s}(c)=v_{e}(c), \quad \phi_{s}(c)=\phi_{e}(c), \quad M_{s}(c)=M_{e}(c), \quad T_{s}(c)=T_{e}(c), \quad w_{s}(c)=w_{e}(c), \quad N_{s}(c)=N_{e}(c) \\
& \phi_{e}(l-a)=0, \quad T_{e}(l-a)=0, \quad w_{e}(l-a)=0
\end{aligned}
$$

yields the integration constants for stage 3 , whose analytical expressions are given in the Appendix. 


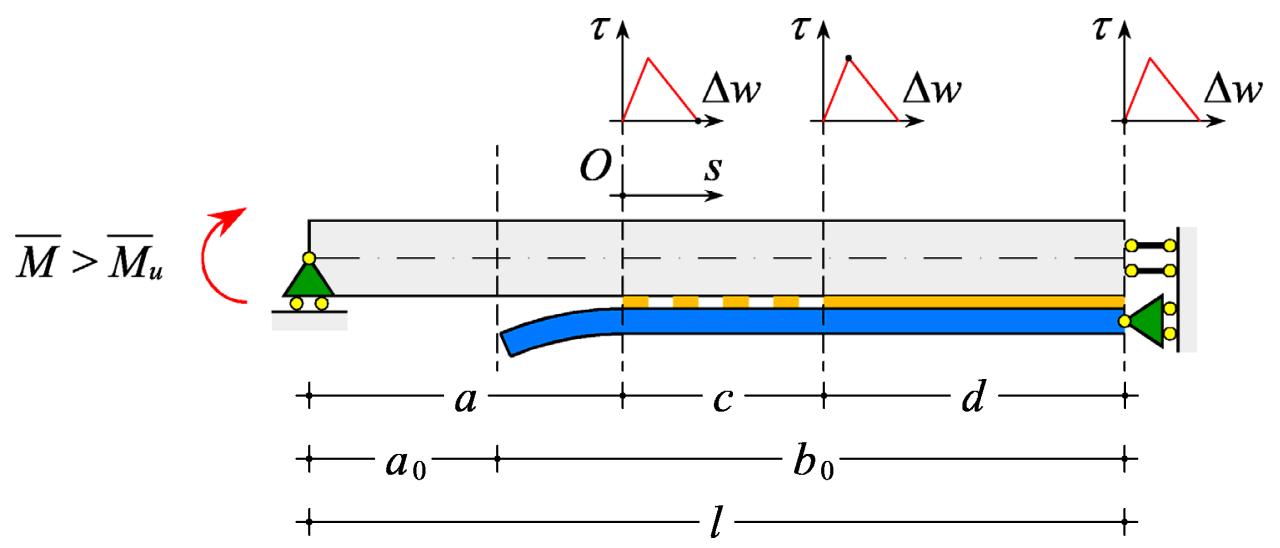

Figure 6: Stage 3) Elastic-Damaged-Deboned interface.

The unknown lengths $a$ and $c$ can both be determined by requiring that the value of the relative displacement at the reinforcement strip's extremity be that corresponding to incipient debonding of the interface and that the relative displacement in the transition section between the damaged and the elastic interface portions be equal to the value corresponding to the elastic limit, or in other terms, that

$$
\Delta w(0)=\Delta w_{u} \quad \text { and } \quad \Delta w(c)=\Delta w_{0}
$$

By substituting the expressions for the relative displacements into Eqs. (39), with the expressions for the integration constants listed in the Appendix, after some simplifications, we obtain the following equation set:

$$
\left\{\begin{array}{l}
{\left[\alpha \beta \sin \beta c+\beta^{2} \cos \beta c \tanh \alpha(l-a-c)\right] \bar{M}-\left(\alpha^{2}+\beta^{2}\right) \bar{M}_{0} \tanh \alpha b_{0}=0} \\
\beta \bar{M} \tanh \alpha(l-a-c)-[\alpha \sin \beta c \tanh \alpha(l-a-c)+\beta \cos \beta c] \bar{M}_{0} \tanh \alpha b_{0}=0
\end{array}\right.
$$

By solving the first of Eqs. (40) for $\tanh \alpha(l-a-c)$ and substituting into the second equation, we get a quadratic equation for $\bar{M}$ :

$$
\bar{M}^{2}+\bar{M} \frac{\alpha}{\beta}\left[\left(1+\frac{\beta^{2}}{\alpha^{2}}\right) \sin \beta c-\frac{1}{\sin \beta c}\right] \bar{M}_{0} \tanh \alpha b_{0}+\left(1+\frac{\alpha^{2}}{\beta^{2}}\right) \bar{M}_{0}^{2} \tanh ^{2} \alpha b_{0}=0
$$

Eq. (41) has two possible solutions, one of which is however to be discarded, as it leads to physically unacceptable values of length $a$. Consistently, we find

$$
\bar{M}=\frac{\alpha}{\beta} \frac{1}{\sin \beta c} \bar{M}_{0} \tanh \alpha b_{0}
$$

and

$$
a=l-c-\frac{1}{\alpha} \operatorname{arctanh}\left(\frac{\beta}{\alpha} \tan \beta c\right)
$$

Since, for physical reasons, length $a$ can only increase, we may consider its derivative with respect to $c$. Thus, it can be seen that as long as $a$ is increasing, during stage 3 of behaviour $c$ must necessarily decrease from the value $c_{u}$ to 0 . At the same time, $a$ grows from the value $a_{0}$ to $l$, that is, until complete detachment of the reinforcement. Actually, such situation is arrived at only asymptotically. In fact, from Eqs. (42) and (43) it can be seen that as $c$ falls towards 0 , length $a$ tends to $l$ and the applied couple $\bar{M}$ increases boundlessly.

\section{Stage 4) Entirely debonded interface}

In stage 4 of behaviour, which is however reached only asymptotically for $\bar{M} \rightarrow \infty$, the interface is entirely debonded and the reinforcement is completely detached from the beam except for the mid-span section where, for reasons of symmetry, 
the relative displacement at the interface and the corresponding tangential stress are always null. The system thus behaves as a beam lacking any reinforcement (Fig. 7). The solution to the problem in this case is given by Eqs. (22)-(25). By imposing the boundary conditions,

$$
\begin{array}{ll}
v_{d}(-l)=0, & M_{d}(-l)=\bar{M} \\
\phi_{d}(0)=0, & T_{d}(0)=0
\end{array}
$$

we deduce the integration constants for this stage:

$$
C_{1}=0, \quad C_{2}=-\frac{\bar{M}}{2 E J}, \quad C_{3}=\frac{\bar{M} l}{E J}, \quad C_{4}=0
$$

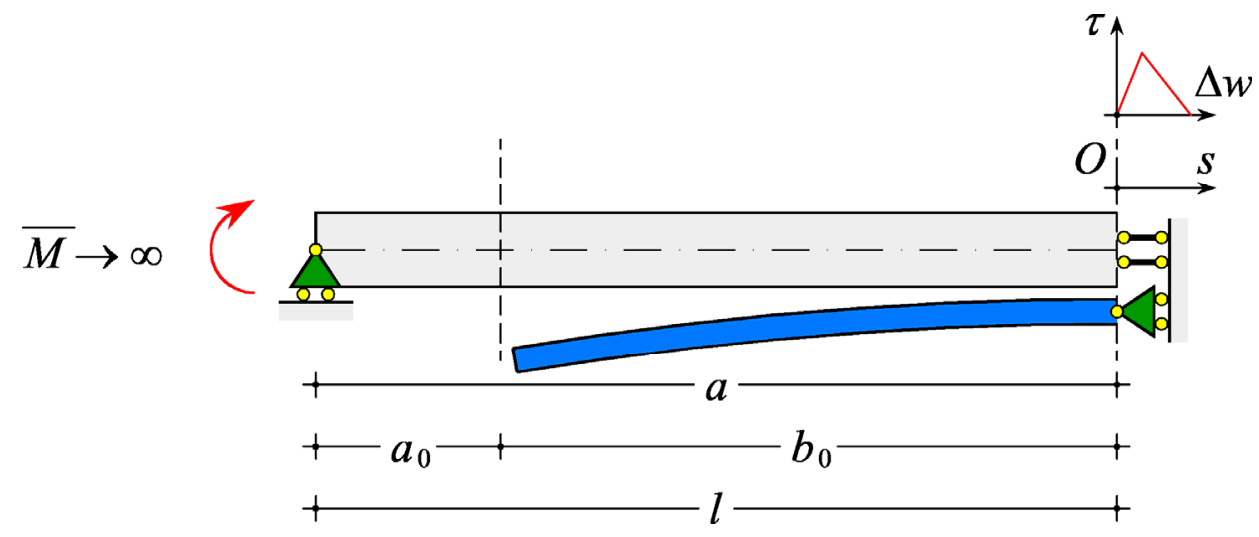

Figure 7: Stage 4) Entirely debonded interface.

\section{NUMERICAL APPLICATION}

$\mathrm{B}$ y way of example, let us consider a concrete beam of length $L=6 \mathrm{~m}$ with rectangular cross section of dimensions $B=30 \mathrm{~cm}$ and $H=40 \mathrm{~cm}$. The width and thickness of the FRP reinforcement are respectively $B_{f}=25 \mathrm{~cm}$ and $t_{f}=0.5 \mathrm{~cm}$. A segment of length $a_{0}=50 \mathrm{~cm}$ is initially devoid of any reinforcement. The other relevant lengths are $l=L / 2=300 \mathrm{~cm}$ and $b_{0}=l-a_{0}=250 \mathrm{~cm}$. The Young's modulus of the beam and FRP strip are respectively $E=30000 \mathrm{MPa}$ and $E_{f}=256000 \mathrm{MPa}$, and the constants defining the interface constitutive law are $k=48 \mathrm{~N} / \mathrm{m}^{3}$, $\tau_{0}=4.2 \mathrm{MPa}$, and $\Delta w_{u}=0.53 \mathrm{~mm}$ [7]. From these values, we also deduce $\Delta w_{0}=\tau_{0} / k=0.0875 \mathrm{~mm}$ and $k_{s}=\tau_{0} /\left(\Delta w_{u}-\Delta w_{0}\right)=9.49 \mathrm{~N} / \mathrm{mm}^{2}$.

Using Eq. (28), we determine the value of the applied couple at the elastic limit, $\bar{M}_{0}=144.73 \mathrm{kN} \mathrm{m}$, corresponding to the end of stage 1. Eq. (35) furnishes the value of the characteristic length of the damaged region, $c_{c}=51.3 \mathrm{~cm}$. For the problem in question, the condition $b_{0}>c_{c}$ is thus clearly verified. Numerical solution of Eq. (34) gives the length of the damaged region at the end of stage 2, $c_{u}=37.6 \mathrm{~cm}$ (Fig. 8). From this latter value, through Eq. (32), we calculate the value of the applied couple at the end of stage $2, \bar{M}_{u}=356.21 \mathrm{kN} \mathrm{m}$. In practice, the same results could have been obtained using the approximate expressions given in Eqs. (29), (36), and (37).

In order to show the trends of the computed quantities (displacements, internal forces and interfacial stresses) along the beam, we define a new abscissa, $z$, measuring the distance of the generic cross section from the left-hand support of the beam. The abscissa $z$, different from the abscissa $s$ used to deduce the analytical solution, turns out to be more effective to present the obtained results. Fig. 9 shows a plot of the beam transverse displacement, $v$, as a function of $z$, for four values of the applied couple, $\bar{M}$, corresponding to the four stages of behaviour described in the previous section. 

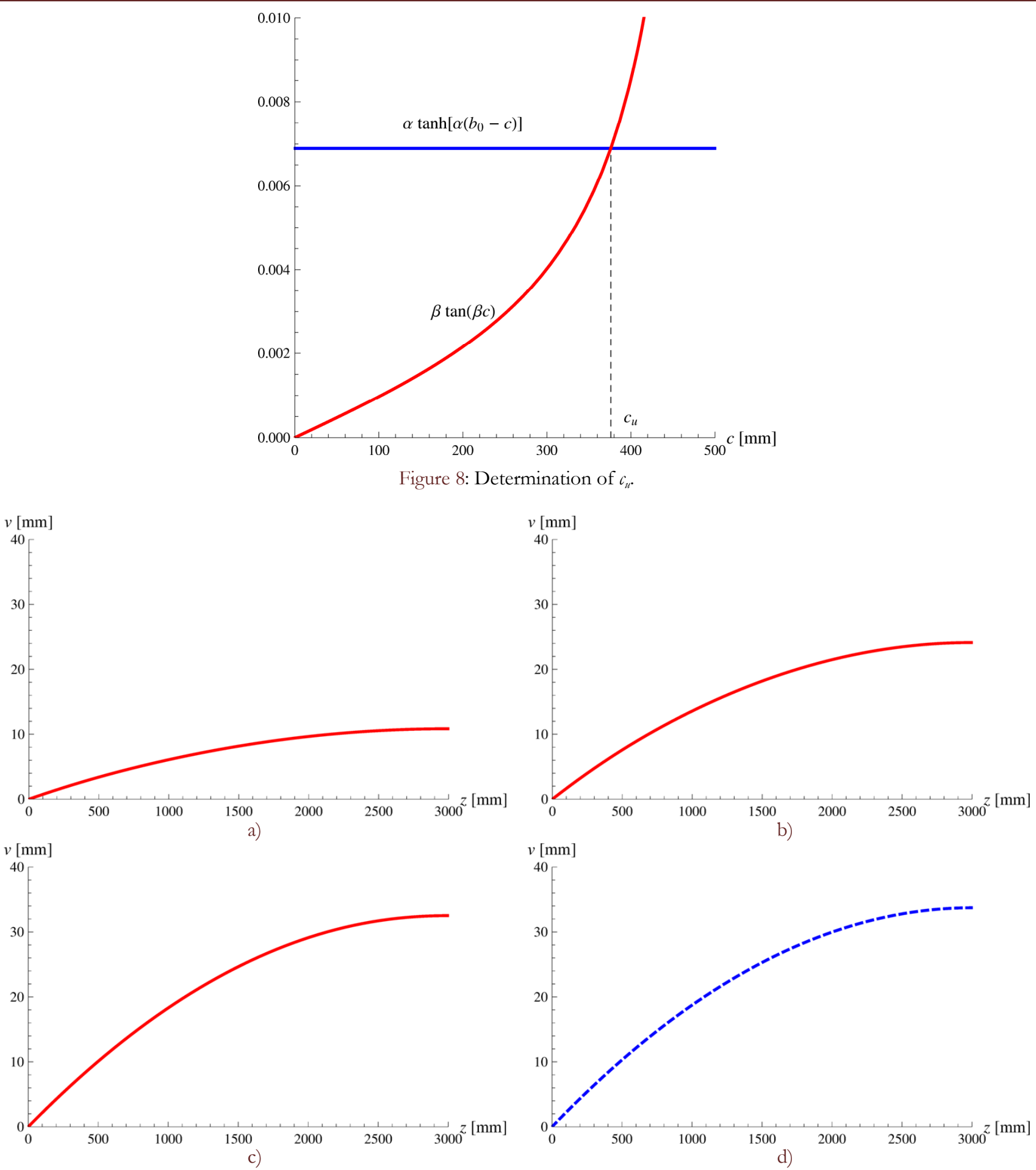

Figure 9: Transverse displacement $v(\mathrm{z})$ : a) stage $1: \quad \bar{M}=\bar{M}_{0} ;$ b) stage $2: \quad \bar{M}=0.9 \bar{M}_{u} ;$ c) stage $3: \quad \bar{M}=1.01 \bar{M}_{u} ;$ d) stage 4 : $\bar{M}=1.01 \bar{M}_{u}$.

Fig. 10 shows a plot of the interfacial relative displacement, $\Delta w$, and tangential stress, $\tau$, as functions of the abscissa, $z$, for three values of the applied couple, $\bar{M}$, corresponding to the first three stages of behaviour described in the previous section. As can be observed, as $\bar{M}$ increases, the relative displacement in the end section of the reinforcement increases: 
the instants such displacement exceeds the values $\Delta w_{0}$ and $\Delta w_{u}$ respectively mark the beginning of interface softening and debonding behaviour.
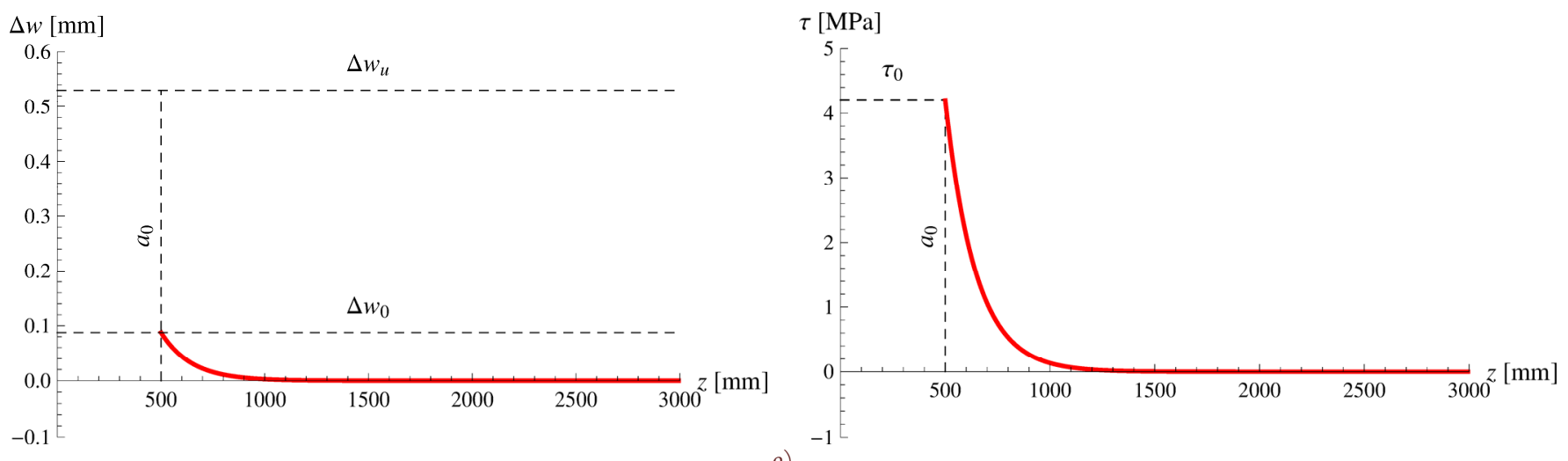

a)
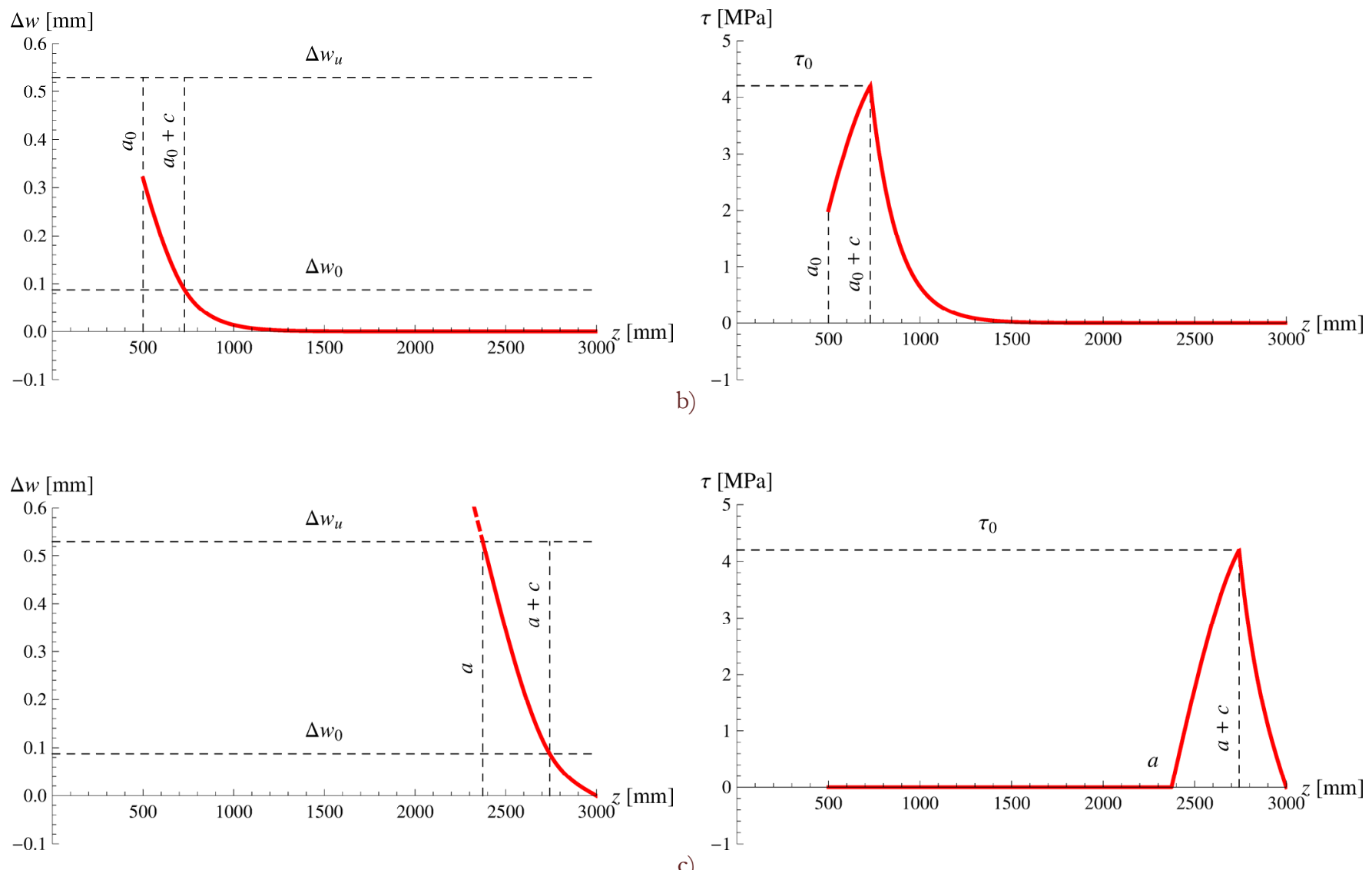

Figure 10: Relative displacement $\Delta w(z)$ and tangential stress $\tau(z):$ a) stage $1: \quad \bar{M}=\bar{M}_{0} ;$ b) stage $2: \quad \bar{M}=0.9 \bar{M}_{u} ;$ c) stage 3 : $\bar{M}=1.01 \bar{M}_{u}$.

Fig. 11 shows a plot of the bending moment in the beam, $M$, as a function of the abscissa, $z$, for four values of the applied couple, $\bar{M}$, corresponding to the four stages of behaviour described in the previous section. Note that with increasing $\bar{M}$ and the consequent progressive detachment of the reinforcement, the strengthened beam's response tends towards that of a beam lacking any reinforcement. 

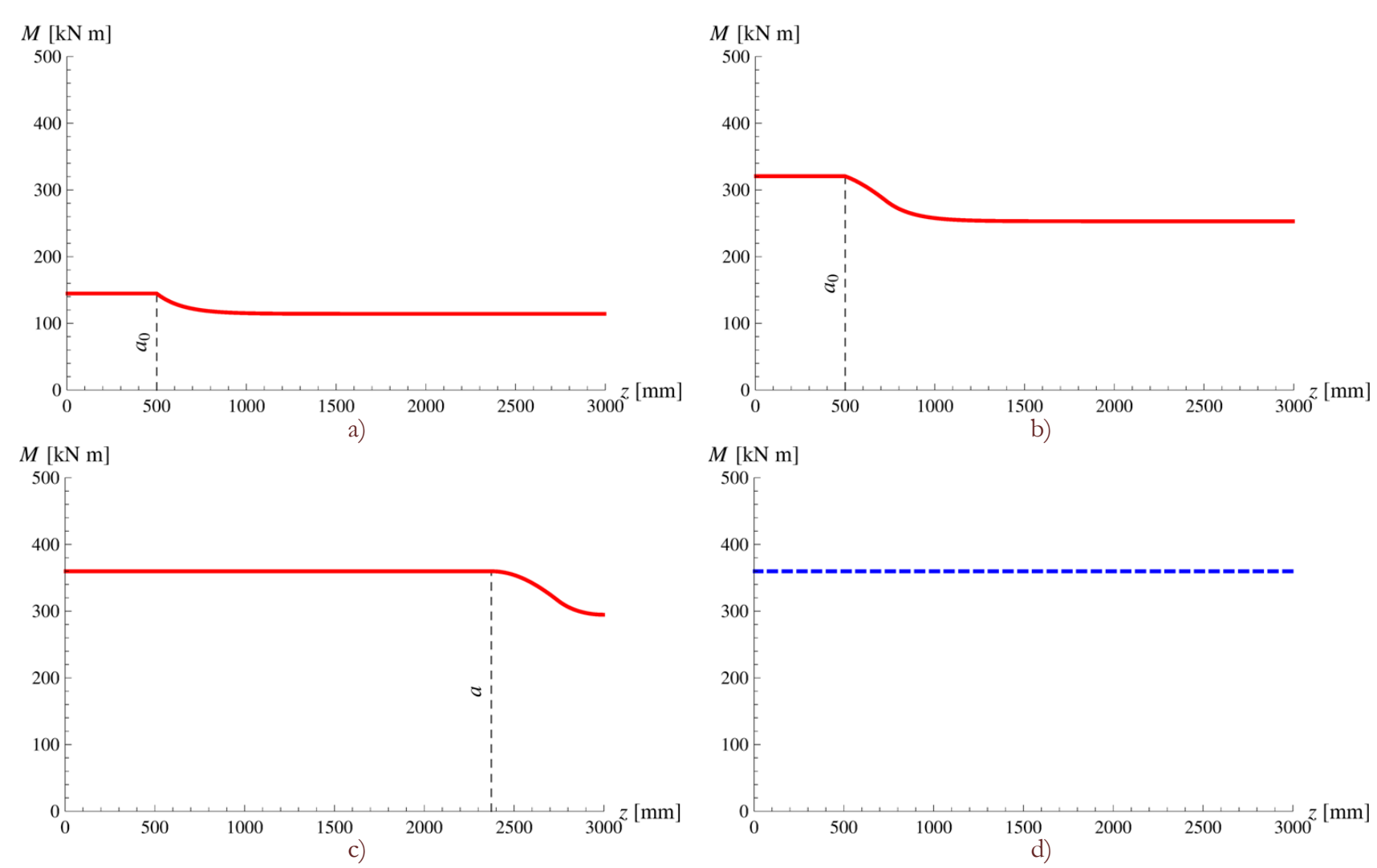

Figure 11: Bending moment $M\left(\right.$ ə): a) stage 1: $\bar{M}=\bar{M}_{0} ;$ b) stage 2: $\bar{M}=0.9 \bar{M}_{u} ;$ c) stage 3: $\bar{M}=1.01 \bar{M}_{u} ;$ d) stage 4: $\bar{M}=1.01 \bar{M}_{u}$.

The shear force in the beam, $T$, is instead null everywhere. This follows from obvious reasons of equilibrium, considering moreover that by virtue of the hypotheses adopted, the FRP reinforcement strip is only able to bear axial stresses.

Fig. 12 shows a plot of the axial force, $N$, on the reinforcement strip as a function of the abscissa, $z$, for three values of the applied couple, $\bar{M}$, corresponding to the first three stages of behaviour described in the previous section. Note that with growing $\bar{M}$ and the consequent progressive detachment of the reinforcement, the length of the active part of the reinforcement is progressively reduced (falling to zero at the limit for $\bar{M} \rightarrow \infty$ ).

Fig. 13 shows the length of the damaged region, $c$, as a function of the length of the region lacking reinforcement, $a$. During stage 2, $c$ increases from 0 to $c_{u}$, while the applied couple goes from $\bar{M}_{0}$ to $\bar{M}_{u}$. During stage 3 , the length $c$ decreases from $c_{u}$ to 0 , while $a$ increases from its initial value, $a_{0}$, up to the half-length of the beam, $l$ (complete detachment of the reinforcement).

Fig. 14 sums up the mechanical response of the FRP-strengthened beam as predicted by the proposed mechanical model. In particular, it shows a plot of the applied couple, $\bar{M}$, as a function of the transverse displacement in the mid-span section, $\delta$. The different stages of behaviour are clearly distinguishable. In stage $1\left(0<\bar{M}<\bar{M}_{0}\right)$, the strengthened beam exhibits a linearly elastic response, while in stage $2\left(\bar{M}_{0}<\bar{M}<\bar{M}_{u}\right)$, the system's response takes on a weakly non-linear quality, due to the fact that a part of the interface has entered the field of softening response (though the non-linearity of such response is not clearly evidenced in the graph of the considered numerical example, it is however evident in the equations describing the model). In stage $3\left(\bar{M}_{u}<\bar{M}\right)$, the response becomes strongly non-linear, due to the fact that increasing portions of the interface enter the debonding range, and the reinforcement accordingly detaches from the beam. In particular, at the beginning of stage 3, an abrupt increase in the mid-span transverse displacement can be observed to occur with almost constant applied load. Subsequently, the behaviour of the system tends asymptotically $(\bar{M} \rightarrow \infty)$ to that of a beam lacking reinforcement, represented in the figure by the dashed blue line (stage 4 ). 

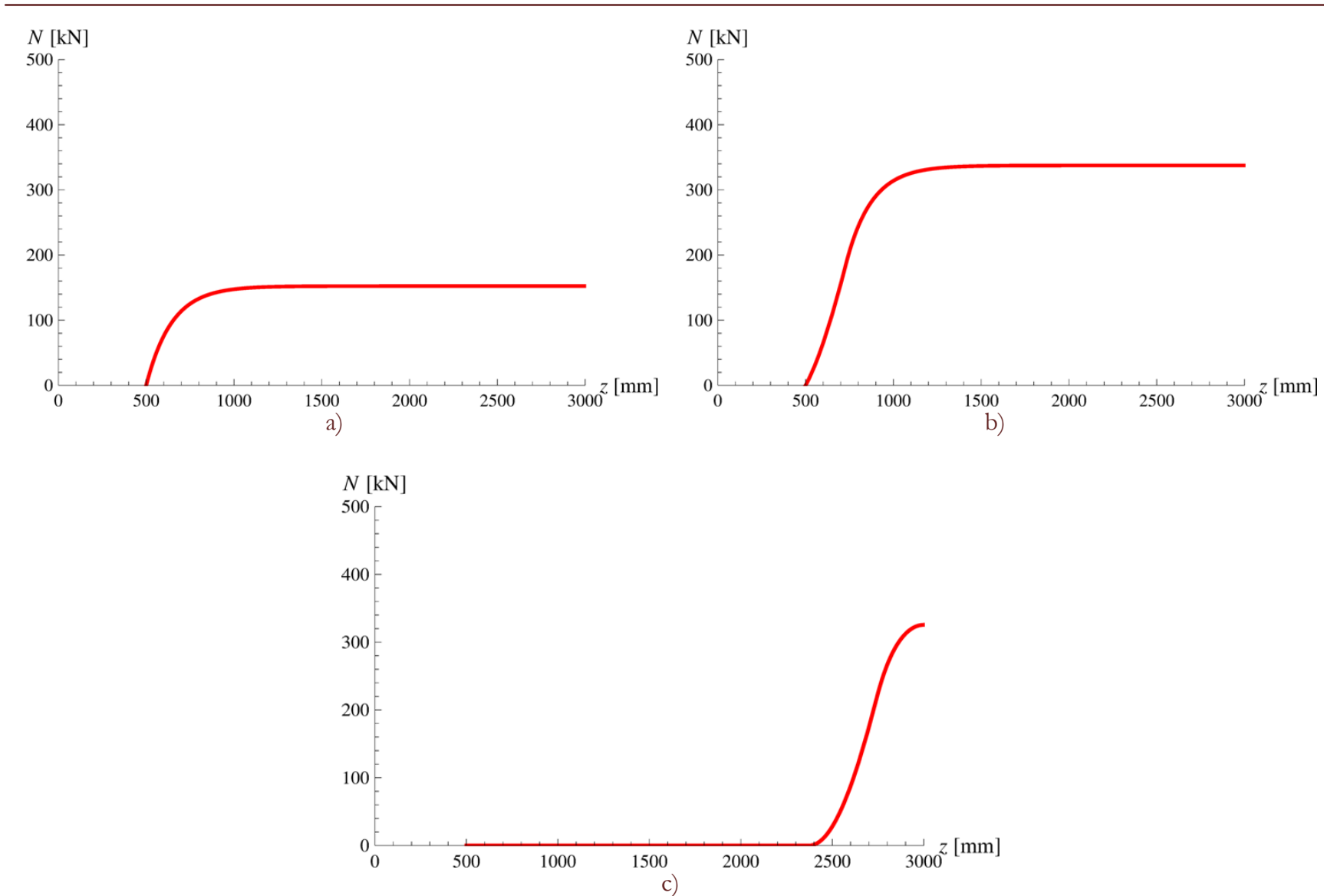

Figure 12: Axial force $N\left(\right.$ s): a) stage 1: $\bar{M}=\bar{M}_{0} ;$ b) stage 2: $\bar{M}=0.9 \bar{M}_{u} ;$ c) stage 3: $\bar{M}=1.01 \bar{M}_{u}$.

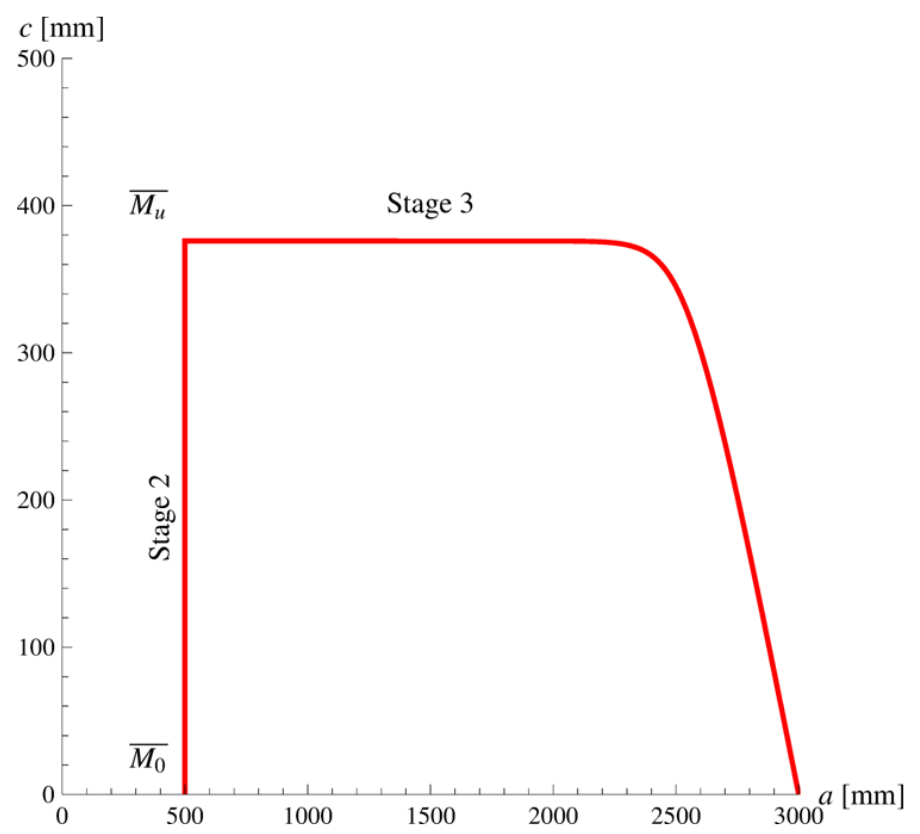

Figure 13 Length of damaged region $c$ as a function of the length, $a$, of the region lacking reinforcement. 


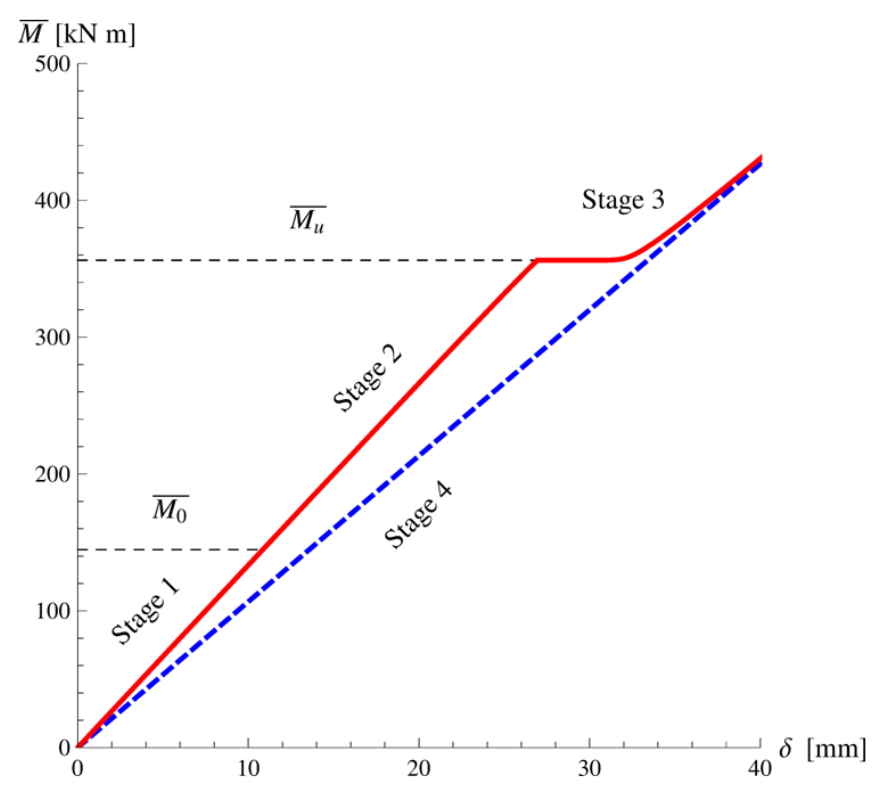

Figure 14: Applied couple $\bar{M}$ as a function of the transverse displacement, $\delta$, of the mid-span section.

Considering the above, it is reasonable to conclude that the value of the applied couple, $\bar{M}_{u}$, corresponding to the end of stage 2 and the beginning of stage 3, represents a limit load for the FRP-strengthened beam. Although the model provides for the possibility of reaching equilibrium at higher load values, this comes at the cost of very large displacements and deformations, together with undesirable dynamic effects, which may not be compatible with the structure's bearing capacity. Moreover, the fact that the stage 2 response may exhibit an only weakly non-linear trend represents a further hazard, as structural failure may come about suddenly without any warning signs.

\section{CONCLUSIONS}

7 he problem of a simply supported beam strengthened with a strip of FRP glued to the intrados and subject to concentrated couples applied to its ends has been analysed. A mechanical model has been developed whereby the beam and reinforcement strip are modelled according to classical beam theory, while the adhesive and adjacent layers are modelled as a zero-thickness interface having a piecewise linear constitutive law defined over three distinct intervals (elastic response - softening response - debonding). The differential problem which describes the model has been solved analytically, and explicit expressions for the main variables of interest (displacements, internal forces, interfacial stresses) have been determined. For such a strengthened beam the model predicts an overall non-linear mechanical response divided into several stages: from a linearly elastic to a non-linear (damaged) response, up to complete detachment of the FRP reinforcement. The values of the applied couple corresponding to the beginning and end of each stage of behaviour have been determined. In particular, approximate, yet effective, analytical expressions for $\bar{M}_{0}$ and $\bar{M}_{u}$ have been given. These values correspond respectively to the end of the linearly elastic response (end of stage 1) and to incipient detachment of the FRP reinforcement (beginning of stage 3). $\bar{M}_{u}$ has been recognised as a limit load for such an FRP-strengthened beam. In fact, higher values of the applied couple produce very large displacements and deformations, potentially incompatible with the structure's bearing capacity. Moreover, it has been noted that these large displacements may occur suddenly, without warning, together with undesired dynamic effects, which could represent a further hazard in implementing strengthening operations of this type.

\section{ACKNOWLEDGEMENTS}

Financial support from MIUR (PRIN 2008 - Prot. N. 20089RJKYN_002) is gratefully acknowledged. 


\section{REFERENCES}

[1] L. Hollaway, M. Leeming, Strengthening of reinforced concrete structures, Woodhead Publishing, UK (1999).

[2] V. Zerbo, A. Di Tommaso, L. Ceriolo, In: Proceedings of structural analysis of historical constructions, Balkema (2004).

[3] L. C. Bank, Composites for Construction, John Wiley \& Sons, New Jersey (2006).

[4] CNR-DT 200/2004, Istruzioni per la Progettazione, l'Esecuzione ed il Controllo di Interventi di Consolidamento Statico mediante l'utilizzo di Compositi Fibrorinforzati, rev. 7 (2008).

[5] J. Yao, J. G. Teng, J. F. Chen, Compos. Part B-Eng., 36 (2005) 99.

[6] C. Mazzotti, M. Savoia, B. Ferracuti, Constr. Build. Mater., 23 (2009) 1529.

[7] A. Cottone, G. Giambanco, Eng. Fract. Mech., 76 (2009) 1957.

[8] P. Cornetti, A. Carpinteri, Eng. Struct., 33 (2011) 1988.

[9] A. Carpinteri, P. Cornetti, N. Pugno, Eng. Struct., 31 (2009) 2436.

[10] L. De Lorenzis, G. Zavarise, Int. J. Solids Struct., 46 (2009) 4181.

\section{APPENDIX}

Stage 1) Entirely elastic interface

Imposing the boundary conditions given in Eqs. (26) yields the following expressions for the integration constants characterising the analytical solution in stage 1:

$$
\begin{aligned}
& A_{1}=-\frac{k B_{f}}{\alpha^{4}} \frac{h^{2}}{E J} \frac{\bar{M}}{E J}, \quad A_{2}=\frac{k B_{f}}{\alpha^{4}} \frac{h^{2}}{E J} \frac{\bar{M}}{E J} \tanh \alpha b_{0}, \quad A_{3}=0 \\
& A_{4}=-\frac{1}{2 \alpha^{2}} \frac{k B_{f}}{E_{f} A_{f}} \frac{\bar{M}}{E J}, \quad A_{5}=\frac{b_{0}}{\alpha^{2}} \frac{k B_{f}}{E_{f} A_{f}} \frac{\bar{M}}{E J} \\
& A_{6}=\frac{k B_{f}}{\alpha^{2}}\left[\frac{a_{0}}{E_{f} A_{f}}\left(\frac{a_{0}}{2}+b_{0}\right)+\frac{h^{2}}{E J}\left(\frac{a_{0}^{2}}{2}+\frac{a_{0}}{\alpha} \tanh \alpha b_{0}+\frac{1}{\alpha^{2}}\right)\right] \frac{\bar{M}}{E J} \\
& C_{1}=0, \quad C_{2}=-\frac{\bar{M}}{2 E J} \\
& C_{3}=\frac{k B_{f}}{\alpha^{2}}\left(\frac{b_{0}}{E_{f} A_{f}}+\frac{h^{2}}{E J} \frac{\tanh \alpha b_{0}}{\alpha}\right) \frac{\bar{M}}{E J} \\
& C_{4}=\frac{k B_{f}}{\alpha^{2}}\left[\frac{a_{0}}{E_{f} A_{f}}\left(\frac{a_{0}}{2}+b_{0}\right)+\frac{h^{2}}{E J}\left(\frac{a_{0}^{2}}{2}+\frac{a_{0}}{\alpha} \tanh \alpha b_{0}\right)\right] \frac{\bar{M}}{E J}
\end{aligned}
$$

Stages 2) Elastic-Damaged interface and 3) Elastic-Damaged-Debonded interface

Imposing the boundary conditions given in Eqs. (30) yields the following expressions for the integration constants characterising the analytical solution in stage 2:

$$
\begin{aligned}
& A_{1}=-\frac{k B_{f}}{\alpha^{4} \beta} \frac{h^{2}}{E J} \frac{\alpha \beta \frac{\bar{M}}{E J}-\left(\alpha^{2}+\beta^{2}\right) \frac{\bar{M}_{0}}{E J} \tanh \alpha b_{0} \sin \beta c}{\alpha \cos \beta c-\beta \tanh \alpha d \sin \beta c} \\
& A_{2}=\frac{k B_{f}}{\alpha^{4} \beta} \frac{h^{2}}{E J} \frac{\alpha \beta \frac{\bar{M}}{E J}-\left(\alpha^{2}+\beta^{2}\right) \frac{\bar{M}_{0}}{E J} \tanh \alpha b_{0} \sin \beta c}{\alpha \cos \beta c-\beta \tanh \alpha d \sin \beta c} \tanh \alpha d \\
& A_{3}=0, \quad A_{4}=-\frac{1}{2 \alpha^{2}} \frac{k B_{f}}{E_{f} A_{f}} \frac{\bar{M}}{E J}, \quad A_{5}=\frac{d}{\alpha^{2}} \frac{k B_{f}}{E_{f} A_{f}} \frac{\bar{M}}{E J}
\end{aligned}
$$




$$
\begin{aligned}
A_{6} & =\frac{k B_{f}}{\alpha^{4} \beta^{3}} \frac{h^{2}}{E J}\left\{\alpha \beta \frac{\alpha^{2}+\beta^{2}+\alpha \beta a_{0}(\alpha \sin \beta c+\beta \tanh \alpha d \cos \beta c)}{\alpha \cos \beta c-\beta \tanh \alpha d \sin \beta c} \frac{\bar{M}}{E J}+\right. \\
& +\alpha^{2} \beta\left(\frac{1}{2} \beta^{2} a_{0}^{2}-1\right) \frac{\bar{M}}{E J}+\frac{\alpha^{2} \beta^{3}}{2 E_{f} A_{f} h^{2}}\left(a_{0}+c\right)(l+d) \bar{M}+ \\
& \left.+\left(\alpha^{2}+\beta^{2}\right)\left[\alpha \beta\left(a_{0}+c\right)-\frac{\alpha^{2} \beta a_{0}+\left(\alpha^{2}+\beta^{2}\right) \sin \beta c}{\alpha \cos \beta c-\beta \tanh \alpha d \sin \beta c}\right] \frac{\bar{M}_{0}}{E J} \tanh \alpha b_{0}\right\} \\
B_{1} & =\frac{k B_{f}}{\alpha^{2} \beta^{3}} \frac{h^{2}}{E J} \frac{\alpha \beta \frac{\bar{M}}{E J}-\left(\alpha^{2}+\beta^{2}\right) \frac{\bar{M}_{0}}{E J} \tanh \alpha b_{0} \sin \beta c}{\alpha \cos \beta c-\beta \tanh \alpha d \sin \beta c} \\
B_{2} & =\frac{k B_{f}}{\alpha^{2} \beta^{3}} \frac{h^{2}}{E J} \frac{\beta^{2} \frac{\bar{M}}{E J} \tanh \alpha d-\left(\alpha^{2}+\beta^{2}\right) \frac{\bar{M}_{0}}{E J} \tanh \alpha b_{0} \cos \beta c}{\alpha \cos \beta c-\beta \tanh \alpha d \sin \beta c} \\
B_{3} & =0, \quad B_{4}=-\frac{1}{2 \alpha^{2}} \frac{k B_{f}}{E_{f} A_{f}} \frac{\bar{M}}{E J} \\
B_{5} & =\frac{k B_{f}}{\alpha^{3} \beta^{2}}\left[\alpha \beta^{2} \frac{d}{E_{f} A_{f}} \frac{\bar{M}}{E J}+\left(\alpha^{2}+\beta^{2}\right) \frac{h^{2}}{E J} \frac{\bar{M}_{0}}{E J} \tanh \alpha b_{0}\right] \\
B_{6} & =A_{1}+A_{6}-B_{1} \\
C_{1} & =0, \quad C_{2}=-\frac{\bar{M}}{2 E J} \\
C_{3} & =\beta B_{1} \sin \beta c+\beta B_{2} \cos \beta c+3 B_{3} c^{2}-2 B_{4} c+B_{5}-3 C_{1} c^{2}+2 C_{2} c \\
C_{4} & =\left(a_{0}+c\right)^{3} C_{1}-\left(a_{0}+c\right)^{2} C_{2}+\left(a_{0}+c\right) C_{3}
\end{aligned}
$$

The integration constants for the solution in stage 3 are given again by Eqs. (A2), provided that the current lengths $a$ and $d=l-a-c$ are considered in place of $a_{0}$ and $d=b_{0}-c$, respectively. 